\title{
Versatile routes to functional RAFT chain transfer agents through the Passerini multicomponent reaction.
}

\author{
Amanda K. Pearce, ${ }^{\mathrm{a}, \mathrm{b} *}$ Alessandra Travanut, ${ }^{\mathrm{a}, \mathrm{b}}$ Benoit Couturaud, ${ }^{\mathrm{a}, \mathrm{b}}$ Vincenzo Taresco, ${ }^{\mathrm{a}}$ Steven \\ M. Howdle, ${ }^{\mathrm{b}, \mathrm{c}}$ Morgan Alexander ${ }^{\mathrm{a}, \mathrm{b}}$ and Cameron Alexander ${ }^{\mathrm{a}, \mathrm{b} *}$. \\ a Molecular Therapeutics and Formulation Division, School of Pharmacy, The University of Nottingham, University \\ Park, NG72RD, Nottingham UK.
}

b EPSRC Programme Grant in Next Generation Biomaterials, School of Pharmacy, The University of Nottingham, University Park, NG72RD, Nottingham UK.

c School of Chemistry, The University of Nottingham, University Park, NG72RD, Nottingham UK

KEYWORDS - Multi-Component Reactions, Controlled Radical Polymerizations, RAFT, Passerini chemistry, Block copolymers.

\begin{abstract}
The widespread adoption of RAFT polymerization stems partly from the ease and utility of installing a functional chain transfer agent onto the ends of the generated polymer chains. In parallel, the Passerini multicomponent reaction offers great versatility in converting a wide range of easily accessible building blocks to functional materials. In this work, we have combined the two approaches such that a single, commonly-available, RAFT agent is used in Passerini reactions to generate a variety of multi-functional RAFT chain transfer agents containing ester linkages. Reactions to generate the multi-functional RAFT agents took place under mild conditions and in good yields. The resulting Passerini-RAFT agents were able to exert control over radical polymerization to generate materials of well-defined molecular weights and dispersity. Furthermore, the presence in these polymer cores of ester and amide functionality through the Passerini chemistries, provided regions in the materials which are inherently biodegradable, facilitating any subsequent biomedical applications. The work overall thus demonstrates a versatile and facile synthetic route to multi functional RAFT chain transfer agents and biodegradable polymers.
\end{abstract}

The RAFT methodology is one of the most commonly used living radical polymerization techniques, due to high versatility in chain transfer agents and availability of a wide variety of compatible monomers. ${ }^{1}$ An additional advantage of RAFT polymerization is that the chain transfer agent (CTA) functionality is retained on the ends of each polymer chain. This phenomenon can be exploited for the derivatization of polymer chains through the use of functional CTAs. ${ }^{2}$ Previous literature has demonstrated efficient strategies for the synthesis of RAFT CTAs, for example the work by Martens et $\mathrm{al}^{3}$ showed the synthesis of functionalized RAFT agents from a single thiolactone precursor, by subjecting the thiolactone to aminolysis with various amine species in a one-pot approach. However, in this case, a full RAFT agent synthesis was required to produce each functionalized RAFT agent, as the different functionalities were introduced through the thiol precursor. Given the diversity of structures which can be constructed by RAFT, ${ }^{4}$ it would be advantageous if there were to be a simple synthetic route which could provide functional chain ends and derivatizable 'handles' in addition to providing a RAFT terminus for further polymerization.
Multicomponent reactions (MCRs), such as the Passerini, Ugi and Biginelli reactions, have been widely explored since their first reports in the $19^{\text {th }}$ century onwards. ${ }^{5}$ These MCRs are characterized by their combination of three or more reactants in one-pot, to form single products in a one-step and mild process. Due to their nature, they possess a high degree of versatility and atom-economy, and have thus been utilized for a diverse range of applications in chemistry, from the synthesis of functionalized cyclic scaffolds to drug discovery and biologically-active compound screening. ${ }^{6}$ More recently however, MCRs have found prominence in the field of polymer chemistry, as a diverse and useful tool for the synthesis of both monomers, polymers and combinatorial libraries of the two. ${ }^{7}$ Particularly, work reported by the groups of $\mathrm{Meier}^{8}$ and $\mathrm{Li}^{9}$ extensively demonstrate the use of the Passerini three-component reaction between an oxo component, an isocyanide, and a nucleophile for postpolymerization functionalization, polycondensation polymerizations and novel monomer syntheses. In addition, the chemistries underlying the Passerini reaction serve to install ester and amide linkages in the polymer backbone, thereby generating sites which can be hydrolytically or enzymatically cleaved. As a consequence, polymers prepared using these routes are of 
great potential interest as biodegradable materials with applications ranging from structural components to medical devices and therapeutics.

In this work, the Passerini multicomponent reaction was used as a facile and versatile method for the introduction of new functionalities onto a pre-existing RAFT chain transfer agent. Through this strategy, functionalized RAFT agents displaying different chemistries could be achieved without the need for synthesizing new RAFT agents each time, by utilizing a carboxylic acid terminated CTA. To our knowledge, this is the first example to demonstrate the combination of RAFT polymerization and the Passerini multicomponent reaction in this way.

In order to demonstrate this concept, the synthesis of new functional RAFT chain transfer agents (CTAs) through the Passerini multicomponent reaction was undertaken using simple and commercially available molecules. This allowed for ease of characterization of the new RAFT agent structures, while still demonstrating the versatility of this approach towards functionalized RAFT agents. Three new CTAs were proposed through Passerini multicomponent reactions. The first was a simple modification to the starting CTA through reaction of the terminal carboxylic acid group to further functionalize the CTA (Figure 1A). For this reaction, benzaldehyde and tert-butyl isocyanide were chosen for the aldehyde and isocyanide functionalities respectively. The reaction was carried out in an equimolar ratio of all components in DCM at $30{ }^{\circ} \mathrm{C}$ for 48 hours, after which the product was purified by column chromatography and characterized. The use of the phenyl and tert-butyl functionalities provided distinct proton NMR signals for the additional components for ease of characterization of the final product. .

A

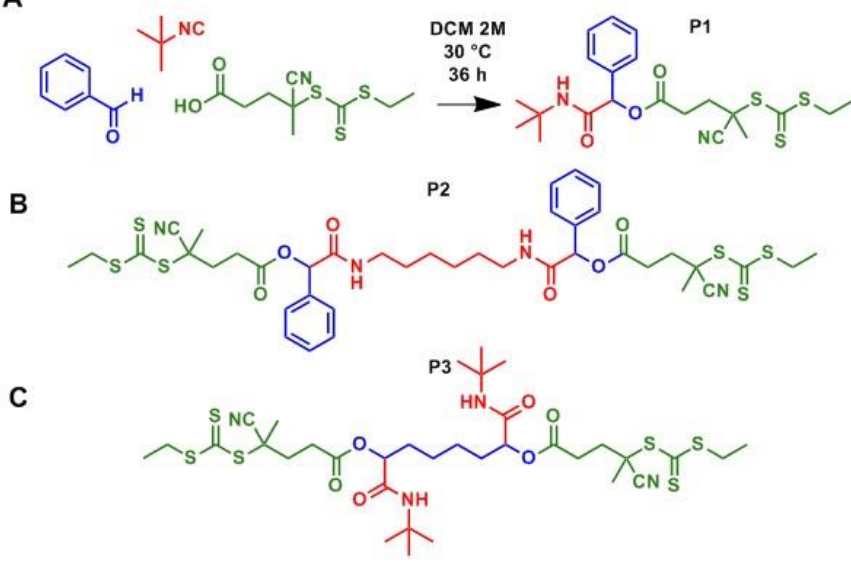

Scheme 1. A) Representative synthetic scheme for the synthesis of the Passerini RAFT agents showing CTA-P1, B) the structure of CTA-P2 and C) the structure of CTA-P 3

The successful synthesis of CTA-P1 was confirmed by NMR, Mass Spectrometry, FT-IR and Elemental Analysis (SI). Following this initial modification of the starting CTA, two further synthetic targets were proposed, CTA-P2 and CTA-P3. These were designed to test the capability of this approach to form more complex, bi-functional RAFT agents. For both of these syntheses, the Passerini reaction was utilized to couple two CTAs together to form a bis-RAFT agent. For CTA-P2, CTA and benzaldehyde were reacted in a $2: 1$ ratio with 1,6-diisocyanohexane in order to achieve a bifunctional RAFT agent with an alkyl linking chain (Figure 1B). This approach was also carried out with the bifunctionality provided by a bis-aldehyde, with CTA-P3 being synthesized from CTA and tert-butyl isocyanide in a 2:1 ratio with hexane-1,6-dial (Figure 1C). The successful synthesis of both of these products demonstrated the feasibility of the Passerini reaction to couple two CTA molecules together through either a bis-isocyanide or a bis-aldehyde, showing an equal reactivity of both functional groups.

Following the synthesis of the three Passerini-modified CTAs, extensive characterization of the purified products was carried out to confirm the proposed structures. The proton and carbon NMR spectra were fully consistent with the expected structures, and mass spectrometry and elemental analysis confirmed the purity of the products. The Passerini reaction brings together an acid, an aldehyde and an isocyanide to produce an amide and an ester bond, with a tertiary carbon situated in between. The formation of these new moieties could be seen in the NMR spectra, as shown in Figure 2, which shows the ${ }^{1} \mathrm{H}$ NMR spectra of CTA-P3 as a representative ${ }^{1} \mathrm{H}$ NMR spectra of the modified CTAs. The peaks at $5.0 \mathrm{ppm}$ (Figure 2, peak f) and $5.7 \mathrm{ppm}$ (Figure 2, peak $\mathrm{n}$ ) were assigned as the proton on the central tertiary carbon and the amide proton that result from a successful Passerini reaction respectively. In the ${ }^{13} \mathrm{C} N M R$ spectra (SI), the signals at 165-172 ppm are typical of carbonyl species, and were assigned to the amide and ester carbonyls generated during the reaction. These, in addition to characteristic signals from the CTA in the purified product, such as the nitrile carbon at $120 \mathrm{ppm}$ and the thiocarbonyl carbon at 218 ppm, verified that the proposed reaction occurred.

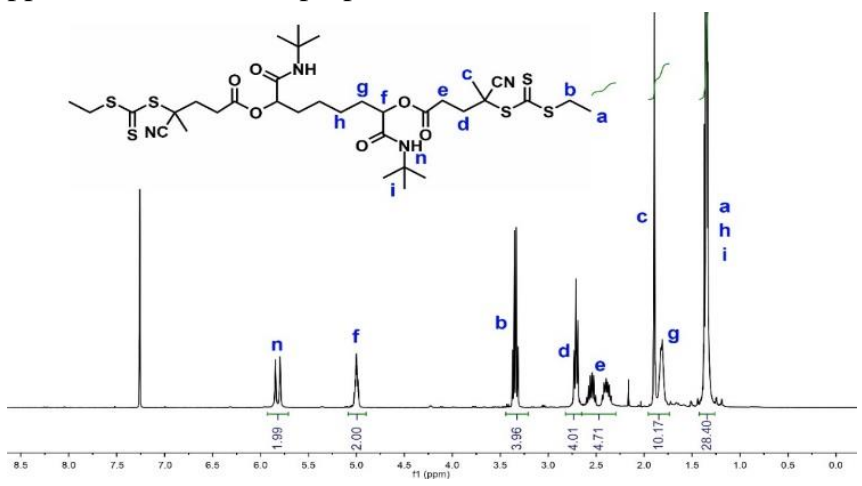

Scheme 2: Representative ${ }^{1} \mathrm{H}$ NMR of the Passerini RAFT agents demonstrating the characterisation of CTA-P 3 . Assignments and integrals confirm the synthesis of the proposed structure.

To confirm these NMR assignments, 2D NMR experiments were performed on all three products. Figure 3 shows the HSQC and HMBC experiments for CTA-P3 as representative spectra. The HSQC experiment was able to confirm a single bond correlation between the carbon signal at $74 \mathrm{ppm}$ and the proton signal at $5.0 \mathrm{ppm}$. The ppm range in both the proton and carbon NMR for these signals agrees with predicted values, and the single bond correlation between the two confirms the assignment in both spectra that these signals are associated with the central tertiary carbon formed by the Passerini reaction. The HMBC experiment further supported these assignments, as well as confirming the identity of the two carbonyl carbon species between $168-170 \mathrm{ppm}$ in the carbon NMR. There was a multiple-bond correlation between the carbon at $168 \mathrm{ppm}$ and the amide proton species at $5.8 \mathrm{ppm}$, indicating the carbon as the carbonyl of the amide bond formed from the Passerini reaction. The carbonyl of the ester bond displayed multiple-bond corre- 
lations between the carbon at $170 \mathrm{ppm}$ and the neighboring proton species at $5.0 \mathrm{ppm}$ (the tertiary carbon) and the ethylene protons from the CEPA RAFT agent $(2.3-2.7 \mathrm{ppm})$. These results, in addition to all other characterization data obtained for the synthesized CTAs, confirmed the modification of the original CTA using this strategy.

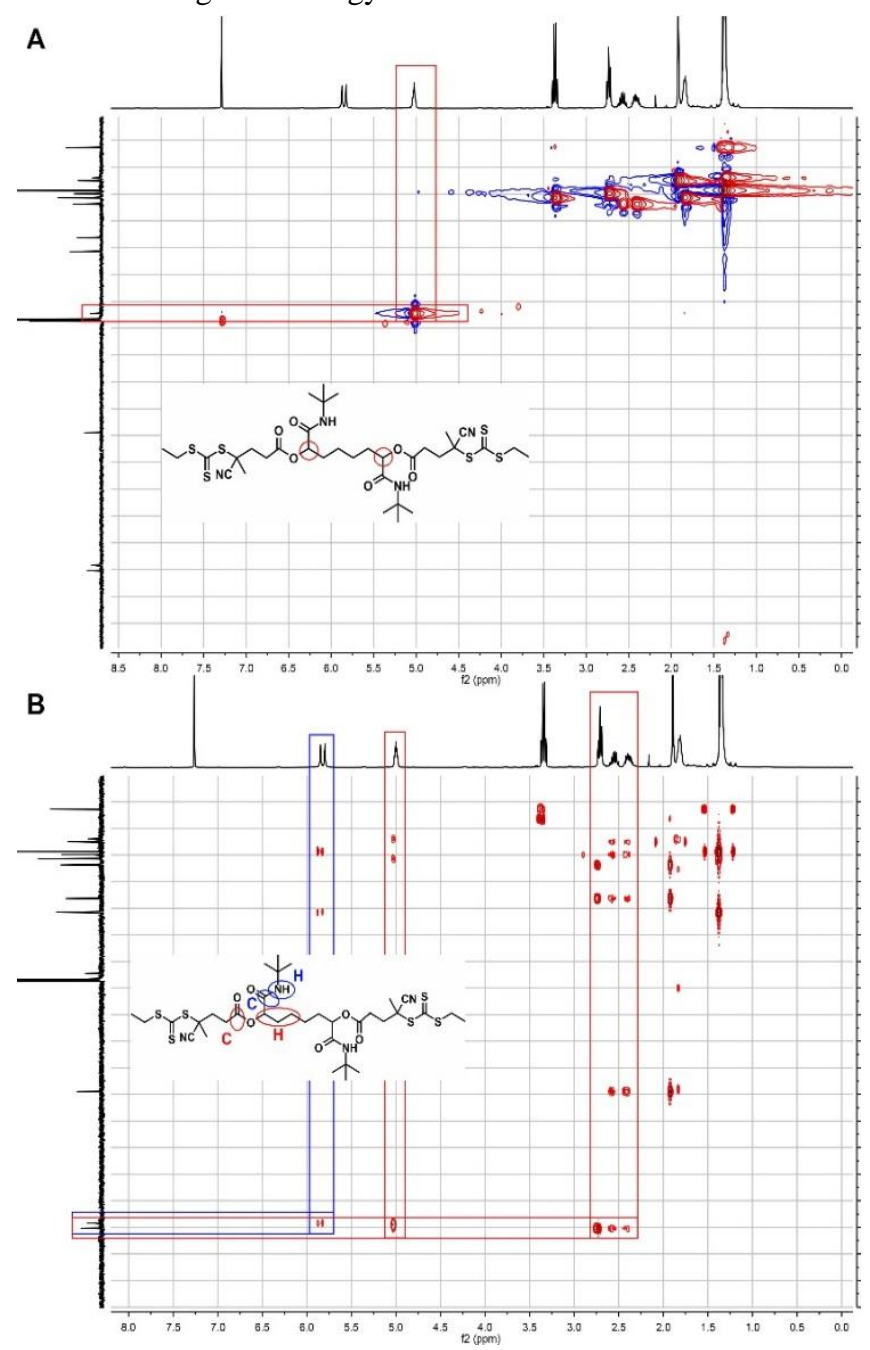

Figure 1. Representative 2D NMR spectra demonstrating the confirmation of peak assignments for CTA-P3. A) HSQC and B) $\mathrm{HMBC}$ with correlations relevant for positive structure confirmation highlighted.

Next, the ability for the modified RAFT agents to control radical polymerizations was investigated, to ensure that the modifications to the original CTA would not significantly interfere with the reaction kinetics or ability for the CTA to control the growth of the propagating polymer species. In order to investigate this, each new CTA, as well as the original unmodified CTA, was used for the homopolymerization of both a hydrophilic monomer (TEGMA) and a hydrophobic monomer (MMA). Each polymerization was conducted for 6 hours, targeting a DP of 25. As shown in Figure 4, each CTA was able to control the homopolymerization of both monomers. All polymers were synthesized to a DP range of 20-27, with dispersities below 1.2

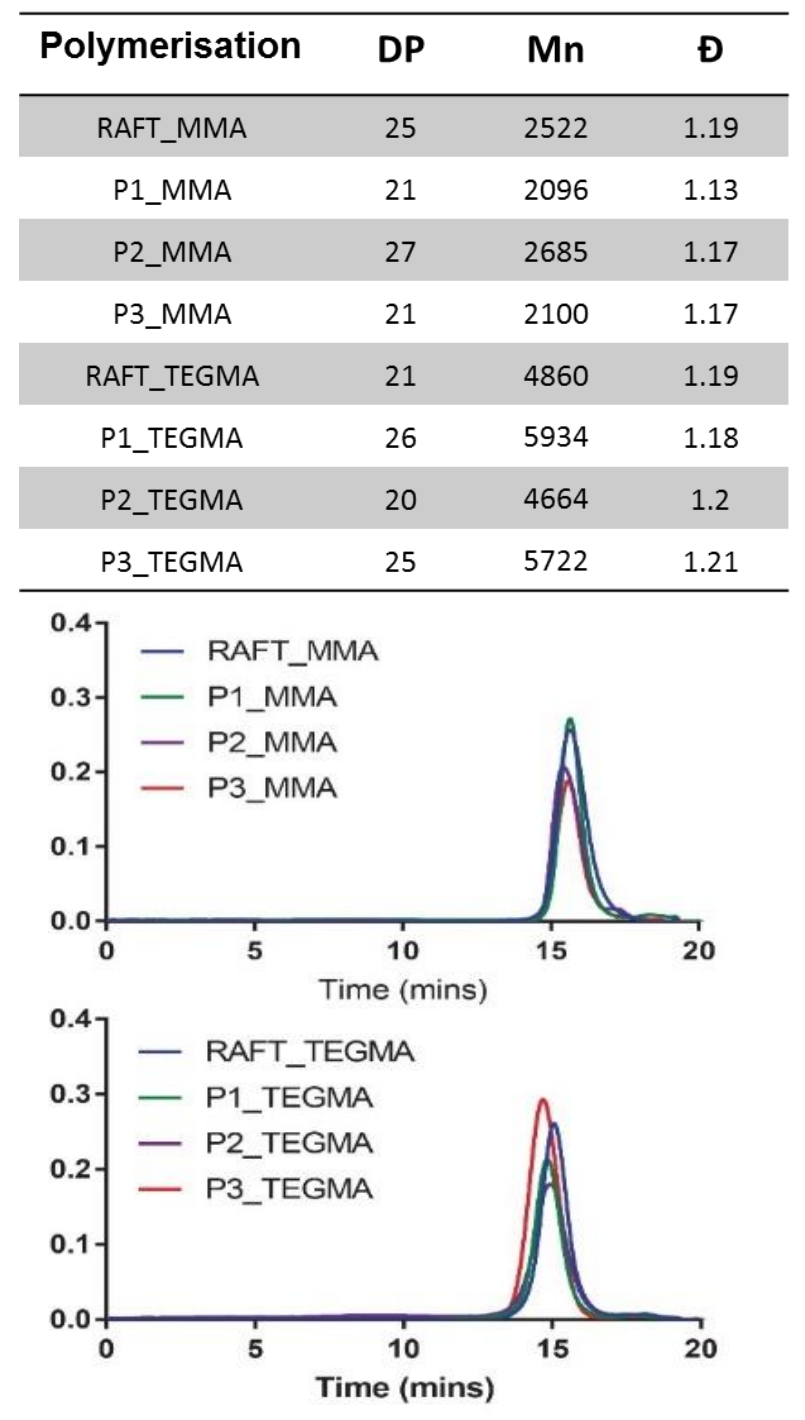

Figure 2. A) Data for each synthesised polymer and B) GPC traces of a) MMA homopolymers and b) TEGMA homopolymers synthesised utilising the Passerini RAFT agents.

The synthesized polymers utilizing CTAs P2 and P3 demonstrated polymerizations could take place with bis-RAFT agents. This meant that the growing chains propagated from both sides of the CTA during the polymerization, however, the GPC data showed that the polymerizations were well controlled, despite having two propagating chains per CTA.

We believe that this strategy for forming CTAs with two or more functional chain transfer groups could prove a useful method for the synthesis of amphiphilic block polymers, simply by changing the components of the Passerini reaction. Additionally, this method could be applied towards the synthesis of star or branched polymers by utilizing the CTA as the core unit. In this short communication we have demonstrated the principle of coupling two orthogonal reaction chemistries to generate small/intermediate size chain transfer agents and methacrylatebased polymers, but the methodology could be adapted to macromonomers and long-chain RAFT agents from Passerini-type oligomers and pre-polymers. 


\section{Conclusions}

In this work, the Passerini reaction was employed as a facile method for the functionalization of RAFT chain transfer agents. This method does not require the synthesis of new individual RAFT agents, but instead allows for the modification of a single CTA to include new functionalities. The Passerini reaction has a wide functional group tolerance, and therefore it is possible to modify the RAFT agents to include a very wide range and combination of functional groups. Additionally, the modified CTAs were able to control the RAFT polymerization of both a hydrophilic and hydrophobic monomer, as well as demonstrating applicability for the synthesis of amphiphilic or branched polymers.

\section{ASSOCIATED CONTENT}

Supporting Information. Spectroscopy data and materials characterization. "This material is available free of charge via the Internet at http://pubs.acs.org.".

\section{AUTHOR INFORMATION}

\section{Corresponding Author}

E-mail: amanda.pearce@nottingham.ac.uk, cameron.alexander@nottingham.ac.uk Tel: +44(o)1158467678.

\section{Author Contributions}

All authors have given approval to the final version of the manuscript.

\section{Funding Sources}

We thank EPSRC for a Programme Grant (EP/Noo6615/1) and the Royal Society (Wolfson Research Merit Award WM150086 to CA and WMXXX to MRA) and the University of Nottingham for funding.

\section{Data access statement}

All raw data created during this research are openly available from the corresponding author (cameron.alexander@nottingham.ac.uk) and at the University of Nottingham Research Data Management Repository (https://rdmc.nottingham.ac.uk/) and all analysed data supporting this study are provided as supplementary information accompanying this paper.

\section{ACKNOWLEDGMENT}

We thank Tom Booth, Cooling, Esme Ireson and Christine Grainger-Boultby for expert technical support.

\section{ABBREVIATIONS}

\section{REFERENCES}

1. (a) Chiefari, J.; Chong, Y.; Ercole, F.; Krstina, J.; Jeffery, J.; Le, T. P.; Mayadunne, R. T.; Meijs, G. F.; Moad, C. L.; Moad, G., Living free-radical polymerization by reversible additionfragmentation chain transfer: the RAFT process. Macromolecules 1998, 31 (16), 5559-5562; (b) Moad, G.; Chong, Y. K.; Postma, A.; Rizzardo, E.; Thang, S. H., Advances in RAFT polymerization: the synthesis of polymers with defined end-groups. Polymer 2005, 46 (19), 8458-8468; (c) Moad, G.; Rizzardo, E.; Thang, S. H., Living Radical Polymerization by the RAFT Process. Australian Journal of Chemistry 2005, 58 (6), 379-410; (d) Perrier, S.; Takolpuckdee, $\mathrm{P}$., Macromolecular design via reversible addition-fragmentation chain transfer (RAFT)/xanthates (MADIX) polymerization.
Journal of Polymer Science Part A: Polymer Chemistry 2005, 43 (22), 5347-5393.

2. (a) Perrier, S.; Takolpuckdee, P.; Westwood, J.; Lewis, D. M., Versatile Chain Transfer Agents for Reversible Addition Fragmentation Chain Transfer (RAFT) Polymerization to Synthesize Functional Polymeric Architectures. Macromolecules 2004, 37 (8), 2709-2717; (b) Bathfield, M.; D'Agosto, F.; Spitz, R.; Charreyre, M.-T.; Delair, T., Versatile Precursors of Functional RAFT Agents. Application to the Synthesis of Bio-Related EndFunctionalized Polymers. Journal of the American Chemical Society 2006, 128 (8), 2546-2547; (c) Skey, J.; O'Reilly, R. K., Facile one pot synthesis of a range of reversible addition-fragmentation chain transfer (RAFT) agents. Chemical Communications 20o8, (35), 4183-4185.

3. Martens, S.; Driessen, F.; Wallyn, S.; Türünç, O.; Du Prez, F. E.; Espeel, P., One-Pot Modular Synthesis of Functionalized RAFT Agents Derived from a Single Thiolactone Precursor. ACS Macro Lett. 2016, 5 (8), 942-945.

4. (a) Noel, A.; Borguet, Y. P.; Wooley, K. L., Self-Reporting Degradable Fluorescent Grafted Copolymer Micelles Derived from Biorenewable Resources. Acs Macro Letters 2015, 4 (6), 645650; (b) Kempe, K.; Ng, S. L.; Noi, K. F.; Muellner, M.; Gunawan, S. T.; Caruso, F., Clickable Poly(2-oxazoline) Architectures for the Fabrication of Low-Fouling Polymer Capsules. Acs Macro Letters 2013, 2 (12), 1069-1072; (c) Roy, D.; Nehilla, B. J.; Lai, J. J.; Stayton, P. S., Stimuli-Responsive Polymer-Antibody Conjugates via RAFT and Tetrafluorophenyl Active Ester Chemistry. Acs Macro Letters 2013, 2 (2), 132-136.

5. (a) Zhu, J.; Bienaymé, H., Multicomponent reactions. John Wiley \& Sons: 2006; (b) Passerini, M., Isonitriles. I. Compound of p-isonitrileazobenzene with acetone and acetic acid. Gazz. Chim. Ital 1921, 51 (2), 126-129.

6. (a) Ruijter, E.; Scheffelaar, R.; Orru, R. V. A., Multicomponent Reaction Design in the Quest for Molecular Complexity and Diversity. Angewandte Chemie International Edition 2011, 50 (28), 6234-6246; (b) Akritopoulou-Zanze, I., Isocyanide-based multicomponent reactions in drug discovery. Current Opinion in Chemical Biology 2008, 12 (3), 324-331; (c) Dömling, A.; Wang, W.; Wang, K., Chemistry and Biology Of Multicomponent Reactions. Chemical Reviews 2012, 112 (6), 30833135; (d) Dömling, A., Recent Developments in Isocyanide Based Multicomponent Reactions in Applied Chemistry. Chemical Reviews 2oo6, 106 (1), 17-89; (e) Sunderhaus, J. D.; Martin, S. F., Applications of Multicomponent Reactions to the Synthesis of Diverse Heterocyclic Scaffolds. Chemistry - A European Journal 2009, 15 (6), 1300-1308; (f) Sunderhaus, J. D.; Dockendorff, C.; Martin, S. F., Applications of multicomponent reactions for the synthesis of diverse heterocyclic scaffolds. Organic letters 2007, 9 (21), 4223-4226; (g) Denmark, S. E.; Fan, Y., The First Catalytic, Asymmetric $\alpha$-Additions of Isocyanides. Lewis-Base-Catalyzed, Enantioselective Passerini-Type Reactions. Journal of the American Chemical Society 2003, 125 (26), 7825-7827.

7. (a) Kakuchi, R., Multicomponent Reactions in Polymer Synthesis. Angewandte Chemie International Edition 2014, 53 (1), 46-48; (b) Rudick, J. G., Innovative macromolecular syntheses via isocyanide multicomponent reactions. Journal of Polymer Science Part A: Polymer Chemistry 2013, 51 (19), 3985-3991.

8. (a) Kreye, O.; Tóth, T.; Meier, M. A. R., Introducing Multicomponent Reactions to Polymer Science: Passerini Reactions of Renewable Monomers. Journal of the American Chemical Society 2011, 133 (6), 1790-1792; (b) Sehlinger, A.; Kreye, O.; Meier, M. A., Tunable polymers obtained from Passerini multicomponent reaction derived acrylate monomers. Macromolecules 2013, 46 (15), 6031-6037; (c) Solleder, S. C.; Meier, M. A., Sequence Control in Polymer Chemistry through the Passerini Three-Component Reaction. Angewandte Chemie International Edition 2014, 53 (3), 711-714. 
9. $\quad$ (a) Deng, X.-X.; Li, L.; Li, Z.-L.; Lv, A.; Du, F.-S.; Li, Z.C., Sequence Regulated Poly(ester-amide)s Based on Passerini Reaction. ACS Macro Lett. 2012, 1 (11), 1300-1303; (b) Wang, Y.-Z.; Deng, X.-X.; Li, L.; Li, Z.-L.; Du, F.-S.; Li, Z.-C., One-pot synthesis of polyamides with various functional side groups via Passerini reaction. Polymer Chemistry 2013, 4 (3), 444-448; (c) Deng, X.-X.; Du, F.-S.; Li, Z.-C., Combination of orthogonal ABB and ABC multicomponent reactions toward efficient divergent synthesis of dendrimers with structural diversity. ACS Macro Lett. 2014, 3 (7), 667-670; (d) Cui, Y.; Zhang, M.; Du, F.-S.; Li, Z.-C., Facile Synthesis of $\mathrm{H}_{2} \mathrm{O}_{2}$-Cleavable Poly(ester-amide)s by Passerini Multicomponent Polymerization. ACS Macro Lett. 2017, 6 (1), 1115 . 
SYNOPSIS TOC (Word Style “SN_Synopsis_TOC

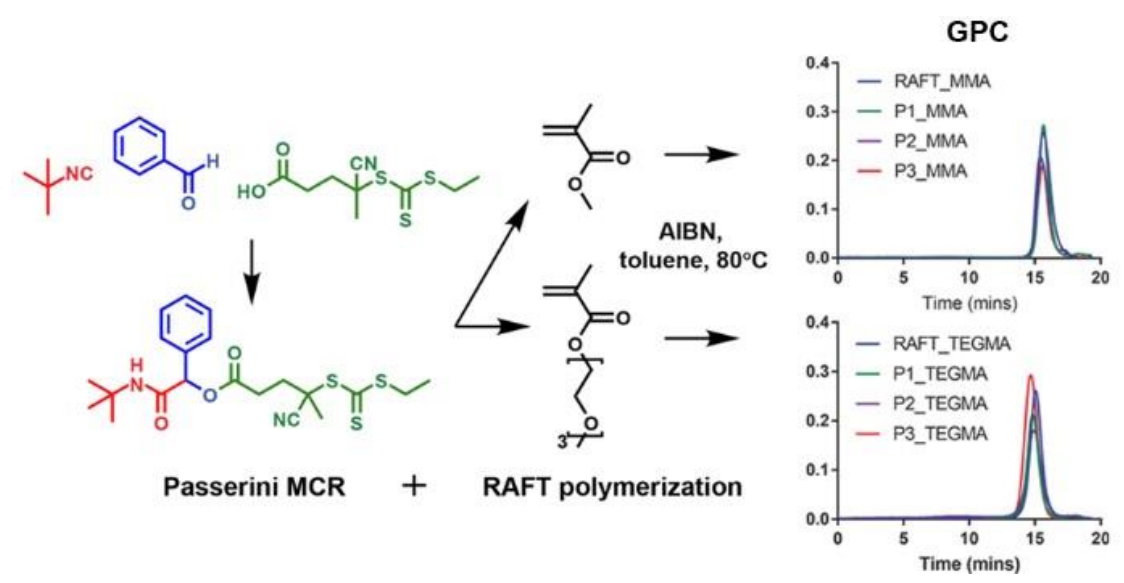

